\title{
Frequency and risk factors associated with dry eye in patients attending a tertiary care ophthalmology center in Mexico City
}

This article was published in the following Dove Press journal:

Clinical Ophthalmology

21 July 2016

Number of times this article has been viewed

\author{
Jaime D Martinez' \\ Anat Galor 2,3 \\ Nallely Ramos-Betancourt \\ Andrés Lisker-Cervantes' \\ Francisco Beltrán' \\ Jorge Ozorno-Zárate' \\ Valeria Sánchez-Huerta' \\ Marco-Antonio Torres- \\ Vera' \\ Everardo Hernández- \\ Quintela' \\ 'Cornea and External Diseases \\ Service, Asociación Para Evitar \\ la Ceguera en Mexico (Association \\ to prevent blindness in Mexico), \\ Universidad Nacional Autónoma \\ de México, Mexico City, Mexico; \\ ${ }^{2}$ Department of Ophthalmology, \\ Miami Veterans Affairs Medical Center, \\ ${ }^{3}$ Cornea and External Diseases \\ Division, Bascom Palmer Eye Institute, \\ University of Miami, Miami, FL, USA
}

Correspondence: Everardo HernándezQuintela

Cornea and External Diseases Services, Asociacion para Evitar la Ceguera en Mexico (Association to prevent blindness in Mexico), \#46 Vicente García Torres, Delegación Coyoacán, México City 04030, Mexico

Tel +52 55 I084 I400

Email evehq@yahoo.com
Purpose: The purpose of this study was to ascertain the frequency and risk factors of dry eye (DE) among patients attending a tertiary care ophthalmology center in Mexico.

Methods: Approximately 338 consecutive new patients attending a tertiary care ophthalmology center in Mexico City underwent an ocular surface examination, which included tear film break-up time, fluorescein corneal staining, Schirmer's test, and evaluation of meibum quality. Symptoms of DE were evaluated by the Ocular Surface Disease Index and Dry Eye Questionnaire-5. Information on demographics, exposures, past medical and ocular history, and medications was also collected.

Results: The frequency of severe DE symptoms was found to be $43 \%$ based on the Ocular Surface Disease Index and 30\% based on Dry Eye Questionnaire-5. Risk factors significantly associated with increased DE symptoms included dry mouth and gastrointestinal ulcer medications. With regard to signs, aqueous tear deficiency was a less-frequent finding $(22 \%)$ in our population than evaporative deficiency (94\%). Risk factors associated with aqueous tear deficiency were dry mouth and diuretic use. No risk factors were associated with evaporative deficiency. Risk factors associated with meibomian gland dysfunction included old age, male sex, arthritis, and use of an antihypertensive. The only risk factor associated with corneal staining was dry mouth.

Conclusion: This is the first study to demonstrate the frequency of symptomatic and clinical DE in a tertiary care ophthalmology center in Mexico. The frequency of DE ranged from 30\% using a symptomatic definition to $94 \%$ using objective measures. Different risk factors were found for different aspects of DE, suggesting differing underlying pathophysiologies behind different DE subtypes.

Keywords: dry eye, meibomian gland dysfunction, ocular surface disease

\section{Introduction}

The Dry Eye Workshop subcommittee (DEWS) defined dry eye (DE) as a "multifactorial disease of the tears and ocular surface that results in symptoms of discomfort, visual disturbance, and tear film instability with potential damage to the ocular surface. It is accompanied by increased osmolality of the tear film and inflammation of the ocular surface". ${ }^{1}$ Based on this definition, it is seen that DE represents a complex process, which involves both symptoms and signs of various diseases. The latter can include decreased tear production, increased tear evaporation, corneal epithelial disruption, and/or meibomian gland changes. The epidemiology of DE has been evaluated in several large population-based studies both in the US and abroad with a wide range of frequencies reported for DE symptoms $(6 \%-50 \%)$, signs $(16 \%-85 \%)$, 
and symptoms plus signs $(73 \%-93 \%) .{ }^{1-14}$ Such different estimates may arise not only due to different ascertainment methodologies but also due to intrinsic differences in the populations studied. For example, the study with a $6 \%$ symptom frequency ascertained symptoms by the presence of any severe DE symptoms through a 7-item questionnaire in a population from Melbourne, Australia, ${ }^{6}$ whereas the study with a $50 \%$ symptom frequency asked about the presence of any positive symptoms through a 6-item questionnaire in Mongolian people in the People's Republic of China. ${ }^{9}$ In a similar manner, the study with an objective frequency of $16 \%$ ascertained DE signs by positive Schirmer's test in Melbourne, Australia, population, whereas the study with $85 \%$ symptom frequency ascertained signs by positive tear film break-up time (TBUT) in elderly Korean population. ${ }^{6,8}$ For example, meibomian gland dysfunction appears more common in Asian countries compared with the USA. ${ }^{15}$ In the present study, the methodology for the diagnosis of DE symptoms and signs was standardized according to the last publication of the DE workshop. ${ }^{16}$

Limited data are available on the frequency and risk factors for DE in Hispanic populations. Data from the Women's Health Study suggest that Hispanic women have more severe DE symptom compared with white women (odds ratio $[\mathrm{OR}]=1.8 ; 95 \%$ confidence interval $[\mathrm{CI}] 1.18-2.80)$, with no information available on the frequency of DE signs. ${ }^{17}$ Two studies specifically investigated the prevalence of DE symptom in Hispanic populations with reported frequencies of $18 \%$ and $25 \%$ in a Spanish population-based study and Hispanic American hospital-based study, respectively. ${ }^{2,18}$ No data are available about the epidemiology of DE in Hispanic populations in Latin America. As such, there is a knowledge gap regarding the frequency of DE in this population, as well as risk factors for the different components of DE, including symptoms and specific signs. This information is important as these data can be used to stratify material and human resources and optimize hospital-based diagnostic algorithms. As such, the purpose of this study was to evaluate the epidemiology of DE in a hospital-based population.

\section{Methods}

\section{Study population}

All new patients who presented to the outpatient clinic of a referral ophthalmology center (Asociación para Evitar la Ceguera) in Mexico City between November 2012 and February 2013 were included in this cross-sectional study. Inclusion criteria were patients were consulting for the first time and were 16 years and older. Exclusion criteria included patients who needed emergency care and who were unable to follow the instructions. The Institutional Review Board and Ethics Committee approval was obtained. The study protocol was approved by the Ethics Committee and the Research Committee of Asociación para Evitar la Ceguera. The research work followed the tenets of the Helsinki Declaration based on the approval by the Institutional Review Board of the hospital and was conducted after obtaining written informed consent from the patients in accordance with institutional guidelines. A total of 350 patients met these criteria between the dates ascertained, and of those, 338 were enrolled into the study. Reasons for nonparticipation of the remaining 12 patients included needing emergency eye surgery $(n=8)$ and mental disabilities $(n=4)$.

\section{Symptom-based DE assessment}

Symptoms of DE were evaluated using two questionnaires that had been validated for Spanish language based on the Mapi Institute guidelines, ${ }^{19}$ the Ocular Surface Disease Index (OSDI), and the Dry Eye Questionnaire-5 (DEQ-5). ${ }^{20,21} \mathrm{We}$ used these two questionnaires to ease the comparisons with prior DE studies. ${ }^{14,22}$ DE symptoms were graded as follows: based on the OSDI score: none (0-12), mild-moderate (13-32), and severe (33-100); and based on the DEQ-5 score: none $(0-5)$, mild-moderate $(6-11)$, and severe $(\geq 12) \cdot{ }^{23,24}$ Risk factor analyses examined which factors were associated with severe DE symptoms by OSDI ( $\geq 33)$ and DEQ-5 $(\geq 12)$.

\section{Objective DE assessment}

Patients underwent a comprehensive examination test sequence following the Dry Eye Workshop subcommittee diagnostic methodology steps. ${ }^{16}$ The measurements included, in the order that they were performed, TBUT ( $4 \mu \mathrm{L}$ of sterile fluorescein placed in conjunctival sac), fluorescein staining (classified by Oxford protocol), ${ }^{16}$ Schirmer's test with anesthesia, ${ }^{16}$ and assessment of meibum quality, rated on a scale of 0-4 (0, clear; 1 , cloudy; 2, granular; 3 , toothpaste type; and 4, no meibum extracted). ${ }^{25}$ Patients were considered to have aqueous tear deficiency if their Schirmer's score was $\leq 5$, evaporative tear deficiency if the TBUT was $\leq 5$, and meibomian gland dysfunction if the meibum quality was $\geq 2$. A corneal staining score $\geq 2$ was considered abnormal. In each participant, the eye with worse signs was used for the analysis.

\section{Patient information}

All information was obtained by patient report, which included demographics (age and sex) and environmental 
exposures (occupation [outdoors or indoors], smoking [current smoker or not], and exposure to air conditioning [yes or no]). Information on ocular diagnoses included use of contact lens (CL), previous ocular surgery, and use of topical medication (artificial tears). Information on various medical conditions and complaints (diabetes, arthritis, thyroid problems, self-reported dry mouth, acne, and depression) and systemic medications (antihypertensive drugs, antihistamines, diuretics, gastrointestinal (GI) ulcer medication, and multivitamins) was also collected.

\section{Statistical analysis}

The data were compiled and subjected to statistical analysis using SPSS Version 20 (IBM Corporation, Armonk, NY, USA). Descriptive statistics were first applied to the population. Linear regression analyses were used to examine the variability of symptoms by DE signs. Pearson correlations were used to assess correlations between metrics. Chi-square, Fischer exact, and student $t$-tests were used (as appropriate) to compare for differences between the groups. Logistic regression analyses were used to identify risk factors for the different DE metrics (subjective and objective). $P$-values $\leq 0.05$ were considered to be of statistical significance.

\section{Results}

\section{Study population}

A total of 338 consecutive patients were included in the analysis. Their mean (standard deviation) age was 45 (16) years (range, 16-85 years), and 187 (55\%) of 338 patients were women (Table 1). Of note, only a minority of individuals (16\%) had comorbid ocular conditions including Sjögren's syndrome $(n=1)$, glaucoma $(n=6)$, use of contact lens

Table I Demographics and dry eye parameters

\begin{tabular}{|c|c|}
\hline Demographics & $\mathbf{N}=338$ \\
\hline Mean age $\pm S D$, range (years) & $45 \pm 16,16-85$ \\
\hline Sex, male, \% (n) & $45 \%(15 I)$ \\
\hline \multicolumn{2}{|l|}{ DE symptoms } \\
\hline OSDI, Mild-moderate (I3-32), \% (n) & $35 \%(118)$ \\
\hline Severe $(\geq 33), \%(n)$ & $43 \%(146)$ \\
\hline DEQ-5, Mild (6-II), \% (n) & $44 \%(148)$ \\
\hline Severe $(\geq 12), \%(n)$ & $30 \%(100)$ \\
\hline \multicolumn{2}{|l|}{ DE signs } \\
\hline Aqueous tear deficiency, Schirmer's $\leq 5, \%$ (n) & $22 \%(74)$ \\
\hline Evaporative deficiency, TBUT $\leq 5, \%$ (n) & $94 \%(319)$ \\
\hline MGD, meibum quality $\geq 2, \%(n)$ & $68 \%(228)$ \\
\hline Corneal staining $\geq 2, \%(n)$ & $11 \%(37)$ \\
\hline
\end{tabular}

Abbreviations: $N$, number of participants; $S D$, standard deviation; $D E$, dry eye; OSDI, ocular surface disease index; DEQ-5, Dry eye Questionnaire-5; MGD, meibomian gland dysfunction; TBUT, tear film break-up time. $(n=11)$, and previous ocular surgery $(n=32)$ (cataract surgery $[\mathrm{n}=10]$, pterygium excision $[\mathrm{n}=8]$, laser-assisted in situ keratomileusis surgery $[n=11]$, vitrectomy $[n=2]$, and strabismus surgery $[n=3])$.

\section{Frequency of DE symptoms and associated risk factors}

The two DE questionnaires were moderately correlated ( $r=0.56, P<0.0005)$. Overall, the frequency of any DE symptoms was $78 \%$ by OSDI and $74 \%$ by DEQ-5. More patients reported severe symptoms through OSDI than through DEQ-5 (Table 1). Severe DE symptoms were most frequent in individuals aged $76-85$ years (where 38\%, $n=3 / 8$, had DEQ-5 $\geq 12$ ) followed by individuals aged $46-55$ years $(36 \%, n=26 / 73)$. The groups with the lowest frequency were those aged 66-75 years $(18 \%, n=6 / 34)$; however, these differences were not statistically significant (Figure 1). As has been reported in previous studies, no significant associations were found between symptoms and clinical signs of DE. ${ }^{26}$ In fact, when all the signs of DE were considered, $3 \%$ of the variability in DEQ-5 scores and 5\% of the variability in OSDI scores were explained by tear film parameters (TBUT, corneal staining, Schirmer's, and meibomian gland dysfunction [MGD]).

Frequencies of the studied demographics and comorbidities within the two DE symptom groups (severe DE symptoms versus no severe DE symptoms) are listed in Table 2. While age and sex were not found to correlate with DE symptoms, in our study several risk factors were identified including having a dry mouth (threefold increased risk by OSDI, 95\% CI 1.6-5.5 and 1.9 fold increased by DEQ-5, 95\% CI 1.1-3.5) and use of a GI ulcer medication (2.3 fold increased risk by OSDI, 95\% CI 1.1-5.1). When both dry mouth and GI ulcer medication use were considered in a forward stepwise logistic regression analysis, both the entities remained significantly associated with severe DE symptoms according to OSDI.

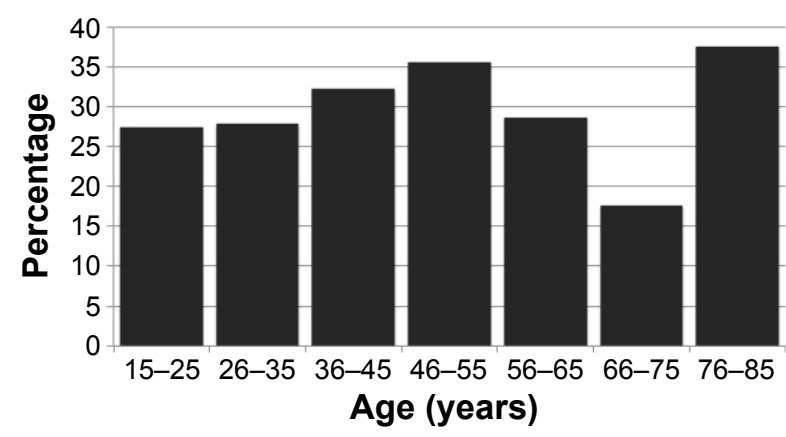

Figure I Percentage of severe dry eye (DE) symptoms (DEQ-5 $\geq 12$ ) by age. Abbreviation: DEQ-5, Dry Eye Questionnaire-5. 
Table 2 Demographics and clinical information by the presence or absence of severe DE symptoms

\begin{tabular}{|c|c|c|c|c|c|c|}
\hline Factors & $\begin{array}{l}\text { Severe DE } \\
\text { symptoms } \\
(\text { OSDI } \geq 33 \text { ) }\end{array}$ & $\begin{array}{l}\text { No severe DE } \\
\text { symptoms } \\
(\text { OSDI <33) }\end{array}$ & $P$-value & $\begin{array}{l}\text { Severe DE } \\
\text { symptoms } \\
(\text { DEQ-5 } \geq I 2)\end{array}$ & $\begin{array}{l}\text { No severe DE } \\
\text { symptoms } \\
(\text { DEQ-5 < I2) }\end{array}$ & $P$-value \\
\hline \multicolumn{7}{|l|}{ Demographics } \\
\hline Age, mean (SD) (N=338) & $46(16)$ & $44(16)$ & 0.3 & $44(15)$ & $45(17)$ & 0.7 \\
\hline Sex, female, \% (n) & $44 \%(83)$ & $56 \%(104)$ & 0.6 & $62 \%(62)$ & $53 \%(125)$ & 0.08 \\
\hline \multicolumn{7}{|l|}{ Environmental factors } \\
\hline Occupation, indoors, \% (n) & $36 \%(53)$ & $46 \%(89)$ & 0.6 & $35 \%(35)$ & $45 \%(107)$ & 0.09 \\
\hline Current smoker, \% (n) & $17 \%(25)$ & $16 \%(30)$ & 0.7 & $12 \%(12)$ & $18 \%(43)$ & 0.1 \\
\hline Exposure to air conditioning, \% (n) & $20 \%(29)$ & $16 \%(31)$ & 0.3 & $23 \%(23)$ & $16 \%(37)$ & 0.2 \\
\hline \multicolumn{7}{|l|}{ Ocular diagnosis } \\
\hline Contact lens use, \% (n) & $2 \%(3)$ & $4 \%(8)$ & 0.4 & $3 \%(3)$ & $3 \%(8)$ & 0.5 \\
\hline Previous surgery, \% (n) & $10 \%(15)$ & $7 \%(14)$ & 0.3 & $14 \%(14)$ & $8 \%(18)$ & 0.06 \\
\hline Lubricant eye drops, \% (n) & $21 \%(3 I)$ & $17 \%(33)$ & 0.3 & $27 \%(27)$ & $16 \%(37)$ & 0.01 \\
\hline \multicolumn{7}{|l|}{ Medical conditions } \\
\hline Diabetes, \% (n) & $10 \%(14)$ & $9 \%(17)$ & 0.8 & $5 \%(5)$ & $11 \%(26)$ & 0.09 \\
\hline Arthritis, \% (n) & $5 \%(7)$ & $5 \%(9)$ & 0.9 & $4 \%(4)$ & $5 \%(I 2)$ & 0.8 \\
\hline Thyroid problems, \% (n) & $4 \%(6)$ & $1 \%(2)$ & 0.08 & $4 \%(4)$ & $2 \%(4)$ & 0.2 \\
\hline Dry mouth, \% (n) & $25 \%(36)$ & $10 \%(19)$ & 0.0005 & $23 \%(23)$ & $13 \%(32)$ & 0.03 \\
\hline Acne, \% (n) & $8 \%(I I)$ & $5 \%(9)$ & 0.2 & $5 \%(5)$ & $6 \%(15)$ & 0.8 \\
\hline Depression, \% (n) & $4 \%(6)$ & $2 \%(4)$ & 0.2 & $3 \%(3)$ & $3 \%(7)$ & 0.6 \\
\hline \multicolumn{7}{|l|}{ Systemic medications } \\
\hline Antihypertensive, \% (n) & $20 \%(29)$ & $13 \%(24)$ & 0.06 & $16 \%(16)$ & $16 \%(37)$ & 0.5 \\
\hline Antihistamine, \% (n) & $3 \%(5)$ & $2 \%(4)$ & 0.3 & $2 \%(2)$ & $3 \%(7)$ & 0.5 \\
\hline Diuretics, \% (n) & $1 \%(2)$ & $2 \%(4)$ & 0.5 & $1 \%(I)$ & $2 \%(5)$ & 0.5 \\
\hline Gl ulcer medication, \% (n) & $12 \%(18)$ & $6 \%(11)$ & 0.04 & $12 \%(12)$ & $7 \%(17)$ & 0.1 \\
\hline Multivitamins, \% (n) & $10 \%(15)$ & $10 \%(20)$ & 0.9 & $8 \%(8)$ & $11 \%(27)$ & 0.2 \\
\hline
\end{tabular}

Note: Bold values represent statistically significant values $P<0.05$.

Abbreviations: DE, dry eye; SD, standard deviation; N, total number of participants; $n$, number in group; OSDI, ocular surface disease index; DEQ-5, dry eye questionnaire-5; Gl, gastrointestinal.

\section{Frequency of DE signs and associated risk factors}

In total, $94 \%$ of patients had at least one abnormality on clinical examination (Schirmer's, TBUT, MGD, or staining) (Table 1). TBUT abnormalities were most common $(\mathrm{n}=319,94 \%)$, followed by MGD ( $\mathrm{n}=228,68 \%)$, aqueous tear deficiency (ATD) $(n=74,22 \%)$, and corneal staining $(n=37,11 \%)$. Frequencies of the studied demographics and comorbidities within those with and without ATD and tear evaporative deficiency, respectively, are listed in Table 3. A self-reported dry mouth $(\mathrm{OR}=1.9,95 \%$ CI 1.02-3.6) and diuretic use $(\mathrm{OR}=7.4,95 \%$ CI 1.3-41) were the two risk factors identified for ATD. When considering both dry mouth and diuretics in a forward stepwise logistic regression analysis, both the entities remained significantly associated with ATD. None of the factors examined correlated with the presence of evaporative deficiency.

Frequencies of the studied demographics and comorbidities within those with and without MGD and corneal staining, respectively, are listed in Table 4. Risk factors associated with MGD included older age (OR $=1.07,95 \% \mathrm{CI}$ 1.05-1.09, per year), male sex (OR $=1.7,95 \%$ CI 1.04-2.6), arthritis (OR $=7.7,95 \%$ CI 1.001-59), and the use of an anti-hypertensive $(\mathrm{OR}=2.7,95 \% \mathrm{CI} 1.3-5.7)$ (Table 4). Interestingly, use of contact lens was a protective factor for MGD (OR $=0.01,95 \%$ CI 0.02-0.5). When considering all MGD risk factors in a forward stepwise logistic regression analysis, old age (as a risk factor) and use of contact lens (as a protective factor) remained significant in the model. The only risk factor associated with corneal staining was dry mouth $(\mathrm{OR}=2.5,95 \%$ CI $1.1-5.4)$.

\section{Discussion}

This study describes the frequency and risk factors for DE in patients attending a tertiary ophthalmology center in Mexico City. Using our case definitions, we found that approximately $1 / 3$ of patients seen in our clinic reported severe DE symptoms and over $90 \%$ of them had a least one sign of DE. Similar to prior studies, ${ }^{26,27}$ no correlation was found between the symptoms and signs of DE. The discrepancy between the symptoms and signs of the disease may be partially explained by an evolving concept that somatosensory dysfunction may underlie some DE symptoms. ${ }^{28,29}$ In the eye, many events including tear hyperosmolarity, air pollution, 
Table 3 Demographics and clinical information by the presence or absence of aqueous and/or evaporative tear deficiency

\begin{tabular}{|c|c|c|c|c|c|c|}
\hline Factors & $\begin{array}{l}\text { ATD } \\
\text { (Schirmer's } \leq 5)\end{array}$ & $\begin{array}{l}\text { No ATD } \\
(\text { Schirmer's >5) }\end{array}$ & $P$-value & $\begin{array}{l}\text { Evaporative } \\
\text { deficiency } \\
(\text { TBUT } \leq 5)\end{array}$ & $\begin{array}{l}\text { No evaporative } \\
\text { deficiency } \\
(\text { TBUT }>5)\end{array}$ & $P$-value \\
\hline \multicolumn{7}{|l|}{ Demographics } \\
\hline Age, mean $(\mathrm{SD})(\mathrm{N}=338)$ & $46(16)$ & $45(16)$ & 0.9 & $45(16)$ & $40(16)$ & 0.1 \\
\hline Sex, female, \% (n) & $61 \%(46)$ & $54 \%(|4|)$ & 0.1 & $56 \%(179)$ & $42 \%(8)$ & 0.1 \\
\hline \multicolumn{7}{|l|}{ Environmental factors } \\
\hline Occupation, indoors, \% (n) & $43 \%(32)$ & $42 \%(110)$ & 0.4 & $42 \%(134)$ & $42 \%(8)$ & 0.5 \\
\hline Current smoker, \% (n) & $17 \%(13)$ & $16 \%(42)$ & 0.4 & $16 \%(5 \mathrm{I})$ & $21 \%(4)$ & 0.3 \\
\hline Exposure to air conditioning, \% (n) & $21 \%(16)$ & $17 \%(44)$ & 0.2 & $18 \%(56)$ & $21 \%(4)$ & 0.4 \\
\hline \multicolumn{7}{|l|}{ Ocular diagnosis } \\
\hline Contact lens use, \% (n) & $3 \%(2)$ & $3 \%(9)$ & 0.5 & $3 \%(I I)$ & $0 \%(0)$ & 0.5 \\
\hline Previous surgery, \% (n) & $7 \%(5)$ & $9 \%(24)$ & 0.3 & $9 \%(28)$ & $5 \%(I)$ & 0.5 \\
\hline Lubricant eye drops, \% (n) & $19 \%(14)$ & $19 \%(50)$ & 0.5 & $19 \%(60)$ & $21 \%(4)$ & 0.5 \\
\hline \multicolumn{7}{|l|}{ Medical conditions } \\
\hline Diabetes, \% (n) & II\% (8) & $9 \%(23)$ & 0.3 & $9 \%(29)$ & $10 \%(2)$ & 0.5 \\
\hline Arthritis, \% (n) & $4 \%(3)$ & $5 \%(13)$ & 0.5 & $5 \%(16)$ & $0 \%(0)$ & 0.3 \\
\hline Thyroid problems, \% (n) & $3 \%(2)$ & $2 \%(6)$ & 0.5 & $3 \%(8)$ & $0 \%(0)$ & 0.6 \\
\hline Dry mouth, \% (n) & $24 \%(18)$ & $14 \%(37)$ & 0.04 & $17 \%(54)$ & $5 \%(I)$ & 0.1 \\
\hline Acne, $\%(n)$ & $5 \%(4)$ & $6 \%(16)$ & 0.5 & $6 \%(18)$ & $11 \%(2)$ & 0.3 \\
\hline Depression, \% (n) & $1 \%(I)$ & $4 \%(9)$ & 0.3 & $3 \%(10)$ & $0 \%(0)$ & 0.9 \\
\hline \multicolumn{7}{|l|}{ Systemic medications } \\
\hline Antihypertensive, \% (n) & $13 \%(10)$ & $16 \%(43)$ & 0.3 & $16 \%(50)$ & $16 \%(3)$ & 0.5 \\
\hline Antihistamine, \% (n) & $\mathrm{I} \%(\mathrm{I})$ & $3 \%(8)$ & 0.6 & $2 \%(7)$ & $11 \%(2)$ & 0.09 \\
\hline Diuretics, \% (n) & $5 \%(4)$ & $1 \%(2)$ & 0.02 & $2 \%(6)$ & $0 \%(0)$ & 0.7 \\
\hline Gl ulcer medication, \% (n) & $8 \%(6)$ & $9 \%(23)$ & 0.5 & $9 \%(27)$ & $11 \%(2)$ & 0.4 \\
\hline Multivitamins, \% (n) & $11 \%(8)$ & $10 \%(27)$ & 0.5 & $11 \%(34)$ & $5 \%(I)$ & 0.3 \\
\hline
\end{tabular}

Note: Bold values represent statistically significant values $P<0.05$.

Abbreviations: ATD, aqueous tear deficiency; GI, gastrointestinal; TBUT, tear film break-up time; N, total number of participants; n, number in group; SD, standard deviation.

Table 4 Demographics and clinical information by the presence or absence of abnormal meibum and staining according to Oxford scheme

\begin{tabular}{|c|c|c|c|c|c|c|}
\hline Factors & $\begin{array}{l}\text { Abnormal quality } \\
(M G D \geq 2)\end{array}$ & $\begin{array}{l}\text { Normal } \\
\text { quality }\end{array}$ & $P$-value & $\begin{array}{l}\text { Staining } \\
\text { Oxford }(\geq 2)\end{array}$ & $\begin{array}{l}\text { Staining } \\
\text { Oxford }(<2)\end{array}$ & $P$-value \\
\hline \multicolumn{7}{|l|}{ Demographics } \\
\hline Age, mean $(\mathrm{SD})(\mathrm{N}=338)$ & $50(15)$ & $35(14)$ & 0.0001 & $45(16)$ & $48(17)$ & 0.3 \\
\hline Sex, female, \% (n) & $51 \%(117)$ & $64 \%(70)$ & 0.02 & $51 \%(19)$ & $56 \%(168)$ & 0.3 \\
\hline \multicolumn{7}{|l|}{ Environmental factors } \\
\hline Occupation, indoors, \% (n) & $41 \%(93)$ & $45 \%(49)$ & 0.2 & $49 \%(18)$ & $41 \%(124)$ & 0.3 \\
\hline Current smoker, \% (n) & $15 \%(35)$ & $18 \%(20)$ & 0.3 & $24 \%(9)$ & $15 \%(46)$ & 0.1 \\
\hline Exposure to air conditioning, \% (n) & $15 \%(35)$ & $23 \%(25)$ & 0.06 & $19 \%(7)$ & $18 \%(53)$ & 0.4 \\
\hline \multicolumn{7}{|l|}{ Ocular diagnosis } \\
\hline Contact lens use, \% (n) & $1 \%(2)$ & $8 \%(9)$ & $<0.00005$ & $3 \%(1)$ & $3 \%(10)$ & 0.2 \\
\hline Previous surgery, \% (n) & $8 \%(17)$ & $11 \%(12)$ & 0.1 & $14 \%(5)$ & $8 \%(24)$ & 0.2 \\
\hline Lubricant eye drops, \% (n) & $19 \%(44)$ & $18 \%(20)$ & 0.4 & $19 \%(7)$ & $19 \%(57)$ & 0.6 \\
\hline \multicolumn{7}{|l|}{ Medical conditions } \\
\hline Diabetes, \% (n) & II\% (25) & $6 \%(6)$ & 0.07 & II\% (4) & $9 \%(27)$ & 0.4 \\
\hline Arthritis, \% (n) & $7 \%(15)$ & $1 \%(1)$ & 0.02 & $8 \%(3)$ & $4 \%(13)$ & 0.2 \\
\hline Thyroid problems, \% (n) & $3 \%(6)$ & $2 \%(2)$ & 0.4 & $6 \%(2)$ & $2 \%(6)$ & 0.2 \\
\hline Dry mouth, \% (n) & $18 \%(40)$ & $14 \%(15)$ & 0.2 & $30 \%(11)$ & $15 \%(44)$ & 0.02 \\
\hline Acne, \% (n) & $5 \%(\mathrm{II})$ & $8 \%(9)$ & 0.1 & $8 \%(3)$ & $6 \%(17)$ & 0.3 \\
\hline Depression, \% (n) & $4 \%(9)$ & $1 \%(I)$ & 0.1 & $5 \%(2)$ & $3 \%(8)$ & 0.3 \\
\hline \multicolumn{7}{|l|}{ Systemic medications } \\
\hline Antihypertensive, \% (n) & $19 \%(44)$ & $8 \%(9)$ & 0.01 & $19 \%(7)$ & $15 \%(46)$ & 0.4 \\
\hline Antihistamine, \% (n) & $1 \%(3)$ & $6 \%(6)$ & 0.06 & $0 \%(0)$ & $3 \%(9)$ & 0.3 \\
\hline Diuretics, \% (n) & $2 \%(5)$ & $1 \%(I)$ & 0.3 & $0 \%(0)$ & $2 \%(6)$ & 0.5 \\
\hline Gl ulcer medication, \% (n) & $10 \%(23)$ & $6 \%(6)$ & 0.1 & $16 \%(6)$ & $8 \%(23)$ & 0.4 \\
\hline Multivitamins, \% (n) & $12 \%(28)$ & $6 \%(7)$ & 0.06 & $8 \%(3)$ & $11 \%(32)$ & 0.4 \\
\hline
\end{tabular}

Note: Bold values represent statistically significant values $P<0.05$.

Abbreviations: MGD, meibomian gland dysfunction; SD, standard deviation; N, total number of participants; $n$, number in groups; Gl, gastrointestinal. 
and surgery can injure the corneal nerves and trigger maladaptive neuroplasticity and thus neuropathic ocular pain. ${ }^{28,29}$ Neuropathic pain is defined as pathological neuroplasticity of the somatosensory system associated with spontaneous firing of peripheral neurons (peripheral sensitization) and/or higher sensory neurons (central sensitization). ${ }^{30}$ Although not diagnostic of neuropathic pain (which requires direct tests of nerve conduction not available in humans), ocular complaints in some DE patients mirror those found in nonocular neuropathic pain disorders including spontaneous burning pain, hyperalgesia, and allodynia (which in the eye manifest as wind- and light-evoked pain). ${ }^{29,31}$

Supporting the idea that different pathophysiologies may underlie different components of DE, we found that different risk factors were associated with different symptoms and signs of disease and also that patients with self-reported dry mouth had a higher frequency of several DE components including severe symptoms, ATD, and corneal staining. This is not surprising given the known relationship between Sjögren's syndrome and ATD. ${ }^{32}$ Interestingly though, most patients with dry mouth complaints did not have a clinical diagnosis of Sjögren's syndrome, supporting data that the condition may be underdiagnosed in the population. ${ }^{33}$ In addition, our study supports prior work linking GI ulcer medication to DE symptoms, ${ }^{34}$ perhaps through their antihistamine effect. However, dissimilar to prior studies, ${ }^{1,34}$ we did not find a significant association between the use of anti-histamine and DE symptoms.

In our population, DE symptoms were not significantly associated with age, and in fact, those 46-55 years old had the second highest frequency of symptoms. These findings are in line with the studies demonstrating DE symptoms in younger individuals, especially in those with occupational exposures such as video display terminal use. ${ }^{7,18,35}$ For example, Uchino et $\mathrm{al}^{35}$ reported that $>4$ hours of video display terminal use was associated with severe symptoms and clinical diagnosed DE disease. In our population, we did not see a difference in severe DE symptom frequency by indoor versus outdoor work. However, we did not specifically stratify individuals by hours of video display terminal use. In a similar manner, we found that male and female patients were equally likely to report severe DE symptoms. Initially, it was thought that the disease affected women predominantly, but our data adds to the existing literature that men also complain of severe DE symptoms and are frequently found to have the signs of DE. ${ }^{36,37}$ In fact, Galor et $\mathrm{al}^{37}$ reported that $19 \%$ of men in a US Veterans Affairs population carried a diagnosis of DE and $68 \%$ of patients evaluated in her tear parameter study had one or more signs of the disease. ${ }^{26}$

MGD is a common finding in older adults, and our data support prior findings that the frequency of MGD increases with age. ${ }^{15,25,38}$ Our study also found a sex difference in MGD frequency with male patients being more likely to have abnormal meibum quality compared with female patients. This is again supported by the literature as prior studies have found a higher frequency of gland drop, lid margin telangiectasia, and meibomian gland orifice plugging in male patients compared with female patients. ${ }^{39,40}$ One potential explanation for this is that androgen levels are known to decrease in aging males ${ }^{39}$ and low androgen levels have been linked with MGD in a number of different populations. ${ }^{41}$ Unexpectedly, CL use was found to be protective for MGD, in contrast to prior studies, which reported an increased frequency of MGD in CL wearers. ${ }^{15,42,43}$ Other studies, however, have not found differences in MGD by CL use. ${ }^{15,44,45}$

\section{Limitations}

This study must be considered in the setting of its limitations. This study was hospital based, without referral information available for the patients, and therefore the findings cannot be directly extrapolated to the general population or private clinical setting. Furthermore, only some but not all DE signs were measured, and therefore we cannot comment on the epidemiology of these metrics (eg, osmolarity, eyelid vascularity, and ocular surface inflammation) in our population. Furthermore, our study was cross-sectional in nature, and as such, the temporal variability of symptoms and signs of DE in our population is unknown. Finally, the frequencies of several comorbidities (eg, use of contact lens, depression, and use of antidepressant medications) were low in our population, limiting our power to assess the effect of these comorbidities on the various components of DE. Despite these limitations, our study adds to the understanding of the frequency and risk factors for DE in Hispanic populations. Furthermore, we found that different risk factors are associated with different aspects of DE. This information is important for the clinicians as it supports the idea that DE is a heterogeneous condition with different patient populations being at variable risk for different components of disease.

\section{Acknowledgments}

This study was supported by the Department of Veterans Affairs, Veterans Health Administration, Office of Research and Development, Clinical Sciences Research, 
Development's Career Development Award CDA-2-024-10S (Dr Galor), NIH Center Core Grant P30EY014801, Research to Prevent Blindness Unrestricted Grant (Dr Galor), and Veterans Affairs career development award >10,000 USD (Dr Galor). The abstract of this paper was presented at the World Ophthalmology Congress, February 5-9, 2016, as an abstract with interim findings.

\section{Disclosure}

The authors report no conflicts of interest in this work.

\section{References}

1. The epidemiology of dry eye disease: report of the Epidemiology Subcommittee of the International Dry Eye WorkShop (2007). Ocul Surf. 2007;5(2):93-107.

2. Viso E, Rodriguez-Ares MT, Gude F. Prevalence of and associated factors for dry eye in a Spanish adult population (the Salnes Eye Study). Ophthalmic Epidemiol. 2009;16(1):15-21.

3. Uchino M, Nishiwaki Y, Michikawa T, et al. Prevalence and risk factors of dry eye disease in Japan: Koumi study. Ophthalmology. 2011; 118(12):2361-2367.

4. Lin PY, Tsai SY, Cheng CY, Liu JH, Chou P, Hsu WM. Prevalence of dry eye among an elderly Chinese population in Taiwan: the Shihpai Eye Study. Ophthalmology. 2003;110(6):1096-1101.

5. Moss SE, Klein R, Klein BE. Prevalence of and risk factors for dry eye syndrome. Arch Ophthalmol. 2000;118(9):1264-1268.

6. McCarty CA, Bansal AK, Livingston PM, Stanislavsky YL, Taylor HR. The epidemiology of dry eye in Melbourne, Australia. Ophthalmology. 1998;105(6):1114-1119

7. Lee AJ, Lee J, Saw SM, et al. Prevalence and risk factors associated with dry eye symptoms: a population based study in Indonesia. Br J Ophthalmol. 2002;86(12):1347-1351.

8. Han SB, Hyon JY, Woo SJ, Lee JJ, Kim TH, Kim KW. Prevalence of dry eye disease in an elderly Korean population. Arch Ophthal. 2011; 129(5):633-638.

9. Guo B, Lu P, Chen X, Zhang W, Chen R. Prevalence of dry eye disease in Mongolians at high altitude in China: the Henan eye study. Ophthalmic Epidemiol. 2010;17(4):234-241.

10. Chia EM, Mitchell P, Rochtchina E, Lee AJ, Maroun R, Wang JJ. Prevalence and associations of dry eye syndrome in an older population: the Blue Mountains Eye Study. Clin Experiment Ophthalmol. 2003; 31(3):229-232.

11. Bukhari A, Ajlan R, Alsaggaf H. Prevalence of dry eye in the normal population in Jeddah, Saudi Arabia. Orbit. 2009;28(6):392-397.

12. Lekhanont K, Rojanaporn D, Chuck RS, Vongthongsri A. Prevalence of dry eye in Bangkok, Thailand. Cornea. 2006;25(10):1162-1167.

13. Jie Y, Xu L, Wu YY, Jonas JB. Prevalence of dry eye among adult Chinese in the Beijing Eye Study. Eye (Lond). 2009;23(3):688-693.

14. Hashemi H, Khabazkhoob M, Kheirkhah A, et al. Prevalence of dry eye syndrome in an adult population. Clin Experiment Ophthalmol. 2014; 42(3):242-248.

15. Schaumberg DA, Nichols JJ, Papas EB, Tong L, Uchino M, Nichols KK. The international workshop on meibomian gland dysfunction: report of the subcommittee on the epidemiology of, and associated risk factors for, MGD. Invest Ophthalmol Vis Sci. 2011;52(4):1994-2005.

16. Methodologies to diagnose and monitor dry eye disease: report of the Diagnostic Methodology Subcommittee of the International Dry Eye WorkShop (2007). The ocular surface. 2007;5(2):108-152.

17. Schaumberg DA, Sullivan DA, Buring JE, Dana MR. Prevalence of dry eye syndrome among US women. Am J Ophthalmol. 2003;136(2): 318-326.

18. Hom M, De Land P. Prevalence and severity of symptomatic dry eyes in Hispanics. Optom Vis Sci. 2005;82(3):206-208.
19. Acquadro C, Conway K, Giroudet C, Mear I. Linguistic Validation Manual for Health Outcome Assessments. Lyon, France: Mapi Institute; 2012.

20. Francisco Beltran NRB, Jaime Martinez, Concepcion Santacruz Valdes, Alejandro Babayan, Cecilia Ramírez-Assad, Elsa Mora Juarez, Everardo Hernandez-Quintela, and Mexican Ocular Surface Disease Study Group. Transcultural Validation of Ocular Surface Disease Index (OSDI) questionnaire for Mexican population. Invest Ophthalmol Vis Sci. 2013;54:E-Abstract 6050.

21. Everardo Hernandez-Quintela NRB, Jaime Martinez, Concepcion Santacruz Valdes, Francisco Beltran, Cecilia Ramírez-Assad, Elsa Mora Juarez, Alejandro Babayan, and Mexican Ocular Surface Disease Study Group, MARVO. Transcultural validation of the 5-item Dry Eye Questionnaire (DEQ-5) for Mexican population. Invest Ophthalmol Vis Sci. 2013;54:e-abstract 4362.

22. Gupta N, Prasad I, Jain R, D'Souza P. Estimating the prevalence of dry eye among Indian patients attending a tertiary ophthalmology clinic. Ann Trop Med Parasitol. 2010;104(3):247-255.

23. Chalmers RL, Begley CG, Caffery B. Validation of the 5-Item Dry Eye Questionnaire (DEQ-5): Discrimination across self-assessed severity and aqueous tear deficient dry eye diagnoses. Cont Lens Anterior Eye. 2010; 33(2):55-60.

24. Schiffman RM, Christianson MD, Jacobsen G, Hirsch JD, Reis BL. Reliability and validity of the Ocular Surface Disease Index. Arch Ophthalmol. 2000;118(5):615-621.

25. Tomlinson A, Bron AJ, Korb DR, et al. The international workshop on meibomian gland dysfunction: report of the diagnosis subcommittee. Invest Ophthalmol Vis Sci. 2011;52(4):2006-2049.

26. Galor A, Feuer W, Lee DJ, Florez H, Venincasa VD, Perez VL. Ocular surface parameters in older male veterans. Invest Ophthalmol Vis Sci. 2013;54(2):1426-1433.

27. Schein OD, Tielsch JM, Munoz B, Bandeen-Roche K, West S. Relation between signs and symptoms of dry eye in the elderly. A populationbased perspective. Ophthalmology. 1997;104(9):1395-1401.

28. Galor A, Levitt RC, Felix ER, Martin ER, Sarantopoulos CD. Neuropathic ocular pain: an important yet underevaluated feature of dry eye. Eye (Lond). 2015;29(3):301-312.

29. Rosenthal P, Borsook D. Ocular neuropathic pain. Br J Ophthalmol. 2016;100(1):128-134

30. Costigan M, Scholz J, Woolf CJ. Neuropathic pain: a maladaptive response of the nervous system to damage. Annu Rev Neurosci. 2009; $32: 1-32$.

31. Galor A, Zlotcavitch L, Walter SD, et al. Dry eye symptom severity and persistence are associated with symptoms of neuropathic pain. Br J Ophthalmol. 2015;99(5):665-668.

32. Lee SY, Petznick A, Tong L. Associations of systemic diseases, smoking and contact lens wear with severity of dry eye. Ophthalmic Physiol Opt. 2012;32(6):518-526.

33. Al-Hashimi I. Xerostomia secondary to Sjogren's syndrome in the elderly: recognition and management. Drugs Aging. 2005;22(11):887-899.

34. Schein OD, Hochberg MC, Munoz B, et al. Dry eye and dry mouth in the elderly: a population-based assessment. Archs Intern Med. 1999; 159(12):1359-1363.

35. Uchino M, Schaumberg DA, Dogru M, et al. Prevalence of dry eye disease among Japanese visual display terminal users. Ophthalmology. 2008;115(11):1982-1988.

36. Galor A, Feuer W, Lee DJ, et al. Prevalence and risk factors of dry eye syndrome in a United States veterans affairs population. Am J Ophthalmol. 2011;152(3):377-384.e372.

37. Galor A, Feuer W, Lee DJ, et al. Depression, post-traumatic stress disorder, and dry eye syndrome: a study utilizing the National United States Veterans Affairs Administrative Database. Am J Ophthalmol. 2012;154(2):340-346.e342.

38. Nien CJ, Massei S, Lin G, et al. Effects of age and dysfunction on human meibomian glands. Arch Ophthalmol. 2011;129(4):462-469.

39. Den S, Shimizu K, Ikeda T, Tsubota K, Shimmura S, Shimazaki J. Association between meibomian gland changes and aging, sex, or tear function. Cornea. 2006;25(6):651-655. 
40. Siak JJ, Tong L, Wong WL, et al. Prevalence and risk factors of meibomian gland dysfunction: the Singapore Malay eye study. Cornea. 2012; 31(11):1223-1228.

41. Azcarate PM, Venincasa VD, Feuer W, Stanczyk F, Schally AV, Galor A. Androgen deficiency and dry eye syndrome in the aging male. Invest Ophthalmol Vis Sci. 2014;55(8):5046-5053.

42. Korb DR, Henriquez AS. Meibomian gland dysfunction and contact lens intolerance. J Am Optom Assoc. 1980;51(3):243-251.
43. Ong BL, Larke JR. Meibomian gland dysfunction: some clinical, biochemical and physical observations. Ophthalmic Physiol Opt. 1990; 10(2):144-148.

44. Hom MM, Martinson JR, Knapp LL, Paugh JR. Prevalence of meibomian gland dysfunction. Optom Vis Sci. 1990;67(9):710-712.

45. Ong BL. Relation between contact lens wear and meibomian gland dysfunction. Optom Vis Sci. 1996;73(3):208-210.

\section{Publish your work in this journal}

Clinical Ophthalmology is an international, peer-reviewed journal covering all subspecialties within ophthalmology. Key topics include: Optometry; Visual science; Pharmacology and drug therapy in eye diseases; Basic Sciences; Primary and Secondary eye care; Patient Safety and Quality of Care Improvements. This journal is indexed on

\section{Dovepress}

PubMed Central and CAS, and is the official journal of The Society of Clinical Ophthalmology (SCO). The manuscript management system is completely online and includes a very quick and fair peer-review system, which is all easy to use. Visit http://www.dovepress.com/ testimonials.php to read real quotes from published authors. 